\title{
ESTUDO EXPERIMENTAL ACERCA DA INFLUENCIA DA VIBRAÇÃO SOBRE O TEOR DE UMIDADE RESIDUAL EM MATERIAL SÓLIDO GRANULAR UTILIZANDO PROTÓTI- PO DE PENEIRA VIBRATÓRIA
}

\author{
R. M. SILVA ${ }^{1}$, V. P. BARBOSA ${ }^{1}$, A. L. MENEZES ${ }^{1}$, C. H. ATAÍDE ${ }^{1}$ e R. GEDRAITE ${ }^{1}$ \\ ${ }^{1}$ Universidade Federal de Uberlândia, Faculdade de Engenharia Química
}

\begin{abstract}
RESUMO - Este artigo apresenta uma contribuição acerca da investigação experimental sobre o emprego do adimensional referente à força- $g$ sobre o teor de umidade residual do material sólido retido sobre tela de protótipo de peneira vibratória de abertura 99 microns (175 mesh) a partir de uma suspensão com concentração volumétrica de $1 \%$ a de $3 \%$. O estudo foi conduzido tendo por base o processo tradicionalmente empregado em unidades de peneiramento de instalações de processamento mineral. Foram realizados experimentos utilizando bancada experimental existente para coletar dados de processo referentes ao teor de umidade residual. Os resultados obtidos sugerem que um aumento no valor do adimensional de força- $g$ tende a contribui para diminuir o teor de líquido residual arrastado com o material sólido retido para suspensão mais diluída.
\end{abstract}

\section{INTRODUÇÃO}

A operação de separação solido-liquido é bastante utilizada tanto na indústria de processamento mineral quanto na perfuração de poços de petróleo, quer seja sob o ponto de vista operacional, ambiental e econômico.

As peneiras evoluíram nos últimos anos, de equipamentos menores e mais simples, capazes de processar apenas sólidos mais grosseiros, para concepções bem mais modernas instaladas em diversos segmentos da indústria. Historicamente, a evolução dos projetos de peneiras vibratórias tem permitido o uso de telas mais finas. Este processo evolutivo levou a quatro fases distintas de tecnologia para proporcionar um melhor desempenho de peneiramento. Tais fases podem ser definidas pelo tipo de movimento aplicado pelos motovibradores no material depositado sobre a tela da peneira, conforme a localização e número de motovibradores utilizados: movimento elíptico desbalanceado, circular, linear e elíptico balanceado (AADE, 1999). Atualmente há peneiras que operam com dois tipos de movimentos: o linear e elíptico balanceado que pode ser escolhido pelo operador de acordo com a sua necessidade.

O peneiramento é uma operação unitária de simples execução, entretanto a descrição matemática e entendimento detalhado dessa operação podem não ser triviais (Standish, 1985; Leschonski, 1979). Essas dificuldades baseiam-se no fato que muitas variáveis afetam a operação de uma peneira vibratória típica, tais como: a tela de peneiramento (formato e tamanho das aberturas), a amplitude e frequência de vibração, o ângulo de inclinação da cesta da peneira, massa específica e distribuições de tamanho e forma das partículas a serem peneiradas, a 
viscosidade da suspensão e a quantidade de material alimentado (Jansen \& Glastonbury, 1967; Fowler \& Lim, 1959). Além disso, tem-se as diversas interações entre essas variáveis, fato que concede a operação um grau de complexidade ainda maior. Todas essas características são utilizadas para entender porque ainda não foi desenvolvida uma metodologia geral e eficiente para predição da operação de peneiramento (Liu, 2009).

O efeito de algumas variáveis operacionais sobre o desempenho do peneiramento vibratório tem sido alvo de investigação de diversos autores, tanto na área de mineração quanto na área de perfuração de poços de petróleo e gás. No peneiramento a seco, Fowler \& Lim (1959) investigaram os efeitos da taxa de alimentação, frequência de vibração, ângulo de inclinação e tamanho de abertura da tela sobre a efetividade de uma peneira vibratória. Beeckmans et al. (1985) estudaram o comportamento do diâmetro de corte sobre a influência do ângulo de inclinação, taxa de alimentação, frequência e amplitude de vibração, abertura de tela e massa específica dos sólidos utilizados nos experimentos. Standish et al. (1986) estudaram o efeito da alimentação, ângulo do deck, rotação dos motovibradores, proporção de partículas de tamanho superior à abertura da tela, massa específica e tamanho relativo de partículas sobre a eficiência de peneiramento com uma abordagem cinética. Trumic \& Magdalinovic (2011) também realizaram uma análise cinética de peneiramento de sólidos minerais para avaliar a influência de fatores como dimensões da tela de peneiramento, distribuição de tamanho de partícula, formato dos materiais particulados, a massa inicial de sólidos e massa específica.

O objetivo deste trabalho foi avaliar experimentalmente a influência do adimensional força- $g$ sobre o teor de umidade arrastado juntamente com os sólidos separados em protótipo de peneira vibratória equipado com tela de 175 mesh. Foram testadas suspensões com concentrações volumétricas iguais a $1 \%$ e $3 \%$ de rocha fosfática moída, com granulometria compreendida entre 0,6 e $600 \mu \mathrm{m}$

\section{MATERIAIS E MÉTODOS}

Os experimentos foram realizados na unidade experimental apresentada pela Figura 1. Essa bancada é formada por uma peneira vibratória em escala piloto, equipada com dois motores vibratórios, uma tela de separação e um sistema de controle de força-g (Guerreiro, 2015). Com finalidade de se obter uma melhor agitação e uma alimentação homogênea, equipou-se o tanque de 500 litros de alimentação com um sistema de agitação mecânica. Uma calha instalada ao fim dessa tubulação permite uma melhor distribuição da alimentação sobre a tela. Um tanque de 200 litros foi utilizado para coleta do fluido passante pela peneira. Esse tanque está ligado a uma tubulação que realimenta o tanque de alimentação, entretanto, durante os experimentos, a bomba centrífuga de realimentação permanece desligada para não haver diluição do material alimentado. Para a coleta do material sólido retido, utilizou-se um recipiente de aproximadamente 20 litros de capacidade.

Seus principais componentes são listados a seguir:

1) Tanque de alimentação de 500 litros com agitador;

2) Calha de alimentação;

3) Tela de peneiramento;

4) Balde para coleta do material retido;

5) Tanque de coleta de 200 litros; 
6) Bomba centrífuga;

7) Motores vibratórios;

8) Acelerômetro piezelétrico para medir a vibração da peneira;

9) Placa de aquisição de dados da National Instruments;

10) Inversor de frequência para alterar a rotação dos motores vibratórios.

Figura 1 - Ilustração da unidade experimental utilizada

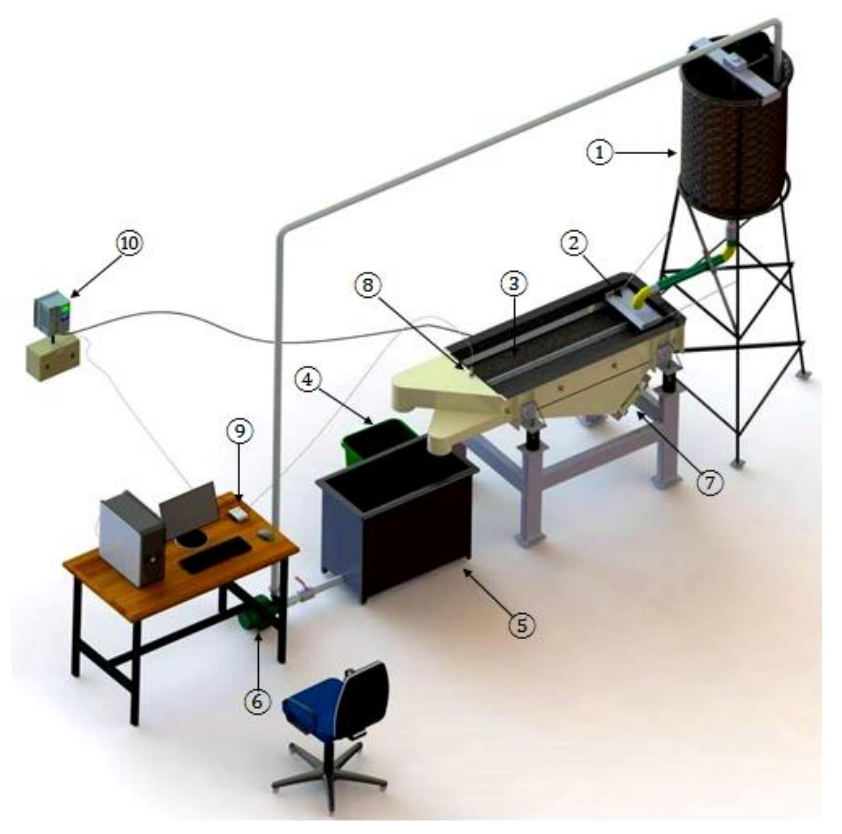

Foram preparados $159 \mathrm{~L}$ de suspensão de rocha fosfática solubilizada em água, nas concentrações de $1 \%, 2 \%$ e $3 \%$ em volume. A alimentação da suspensão foi realizada abrindo-se a válvula manual do tanque principal (1) para alimentar a suspensão sobre uma calha de aço inoxidável visando distribuir o material uniformemente sobre a largura da tela da peneira. A peneira usou tela de 175 mesh, que foi a que apresentou melhor eficiência de separação (Guerreiro, 2015). Depois de esgotada toda a suspensão do tanque de alimentação principal, o material retido era coletado e devidamente pesado em uma balança analítica, e o material passante era recolhido no tanque de coleta. $\mathrm{O}$ valor da massa total de material retido foi usado para a avaliação do teor de umidade residual, sendo separadas três amostras para a avaliação do teor de umidade, realizada por pesagem, após submeter o material coletado à secagem em estufa a $105 \pm 4^{\circ} \mathrm{C}$ por 24 horas.

Após a coleta de amostras para análises, os sólidos utilizados no peneiramento eram manualmente retornados para o tanque principal de alimentação e o líquido passante era retornado através de uma bomba centrífuga localizada na saída do tanque de coleta. Os experimentos foram conduzidos para os valores do adimensional de força-g, impostos à tela da peneira vibratória, variando entre 1,0 e 3,5 e para concentrações volumétricas da suspensão iguais a $1 \%, 2 \%$ e $3 \%$. 


\section{RESULTADOS E DISCUSSÃO}

O comportamento experimental da variável de processo teor de umidade dos sólidos retidos em função da força- $g$ aplicada para a peneira vibratória estudada e considerando a concentração volumétrica de sólidos de $1 \%$ é apresentado na Figura 2, a concentração volumétrica de sólidos de $2 \%$ é apresentado na Figura 3 e a concentração volumétrica de sólidos de $3 \%$ é apresentado na Figura 4. Importante ressaltar que os ensaios foram realizados em triplicata.

Figura 2 - Teor de umidade residual em função do adimensional de força- $g-\mathrm{Cv}=1 \%$

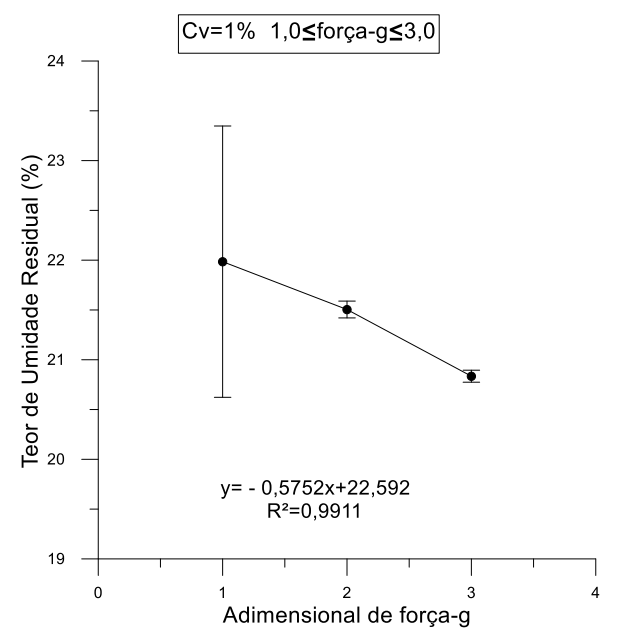

Figura 3- Teor de umidade residual em função do adimensional de força- $g-\mathrm{Cv}=2 \%$

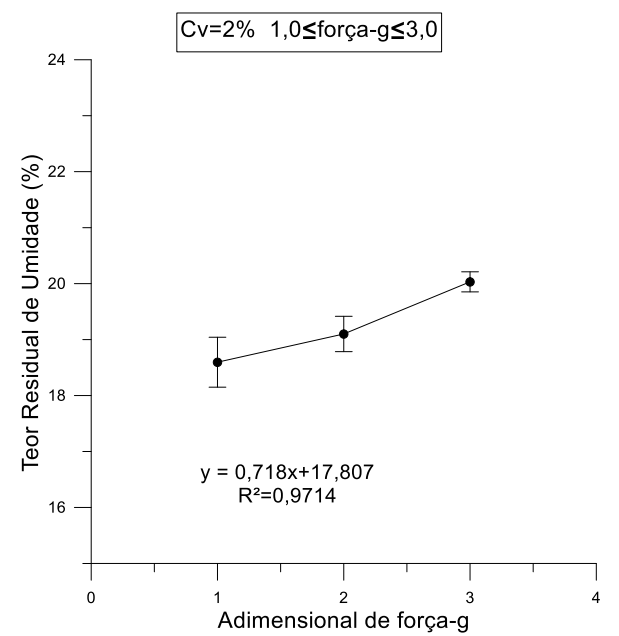

Figura 4 - Teor de umidade residual em função do adimensional de força- $g-\mathrm{Cv}=3 \%$

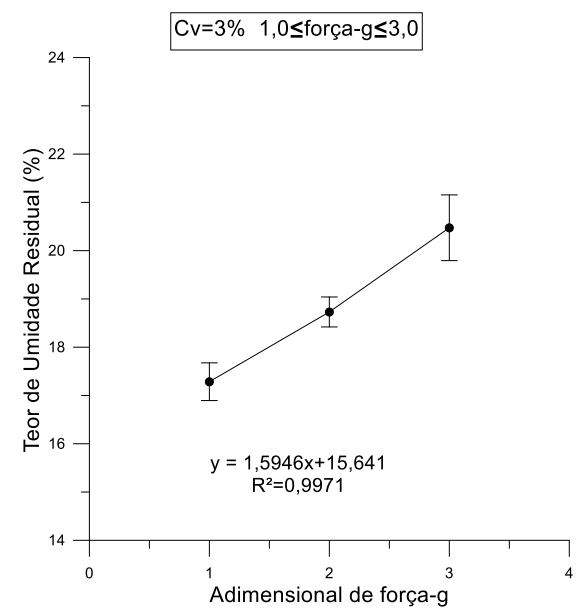

Com base nos resultados obtidos, verificou-se que, para a suspensão alimentada com concentração volumétrica igual a $1 \%$, à medida que o valor do adimensional de força-g foi aumentado, a análise do gráfico apresentado na Figura 2 sugere que o teor de umidade residual apresenta tendência de diminuição para os valores do adimensional de força-g estudados. Contudo, levando em conta o desvio padrão do teor de umidade residual correspondente ao 
ponto do valor de força-g igual a 1,0 pode-se questionar este comportamento, haja vista o fato de que o valor da incerteza associado com as medições foi muito elevado. Uma possível justificativa para este comportamento experimental pode estar associado com o fato de a frequência de vibração ser baixa, não contribuindo de maneira efetiva para o deságue do material solido sobre a tela da peneira e provocando, deste modo, significativa variabilidade nos resultados. Para os valores de força- $g$ iguais a 2,0 e 3,0, o desvio padrão foi bem menor, sugerindo que a influencia da vibração foi mais significativa. Sob o ponto de vista estatístico, contudo, os resultados apresentados na Figura 2 não permitem concluir que o aumento do valor da força-g contribui para diminuir o teor de umidade residual.

Para o caso da suspensão alimentada com concentração volumétrica igual a $2 \%$, à medida que o valor do adimensional de força- $g$ foi aumentado, o teor de umidade residual apresentou a tendência de aumento para os valores do adimensional de força-g estudados. Deve, contudo, ser ressaltado o fato de que o teor de umidade residual foi menor do que o verificado para os testes com concentração volumétrica de $1 \%$, apesar da tendência de aumento apresentada no gráfico da Figura 3. Este comportamento pode ser justificado pelo aumento na velocidade de transporte do material sobre a tela de separação da peneira, uma vez que a vibração mais intensa deveria - de fato - proporcionar um aumento no valor da velocidade de transporte, desfavorecendo deste modo o deságue do material.

Para o caso da suspensão alimentada com concentração volumétrica igual a 3\%, à medida que o valor do adimensional de força- $g$ foi aumentado, o teor de umidade residual apresentou a tendência de aumento para os valores do adimensional de força-g estudados. Deve, contudo, ser ressaltado o fato de que o teor de umidade residual foi menor do que o verificado para os testes com concentração volumétrica de $1 \%$, apesar da tendência de aumento apresentada no gráfico da Figura 4. Valem aqui os mesmos argumentos utilizados para o caso de concentração volumétrica igual a $2 \%$.

\section{CONCLUSÃO}

Considerando os resultados obtidos nos experimentos realizados, pode-se concluir que o teor de umidade residual nas suspensões mais diluídas respondeu de maneira inversa ao aumento do valor da força-g, ao passo que as suspensões mais concentradas responderam de maneira diretamente proporcional ao aumento do valor da força-g aplicada.

Com base nos resultados dos experimentos, pode-se afirmar que os valores obtidos para o teor de umidade residual sugerem que estes apresentaram pouca variação entre si. Este fato aponta para a necessidade de investigar outros valores de força-g e de concentração volumétrica da suspensão alimentada, para avaliar de maneira mais completa mecanismo de remoção da umidade residual.

\section{REFERÊNCIAS BIBLIOGRÁFICAS}

AADE. Shale Shakers and Drilling Fluid Systems. Houston: Gulf Publishing Company, 1999.

BEECKMANS, J. M., GERMAIN, E. R., \& MCINTYRE, A. Performance Characteristics of a Probability Screening Machine. Powder Technology, p. 249-256, 1985. 
FOWLER, R. T., \& LIM, S. C. The Influence of Various Factors upon the Effectiveness of Separation of a Finely Divided Solid by a Vibrating Screen. Chemical Engineering Science, 1959.

GUERREIRO, F. S. Estudo experimental do peneiramento vibratório em batelada de suspensão diluída de rocha fosfática: determinação do teor de umidade de material retido, eficiência granulométrica e diâmetro de corte. 67f. Dissertação (Mestrado em Engenharia Química) - Faculdade de Engenharia Química, Universidade Federal de Uberlândia, Uberlândia, 2015.

HOBEROCK, L. L. Screen Selection is Key to Shale-Shaker Operation. Oil \& Gas Journal, 130-132, 137-141, 1981.

JANSEN, M. L., \& GLASTONBURY, J. R. The Size Separation of Particles by Screening. Powder Technology, p. 334-343, 1967.

LESCHONSKI, K. Sieve analysis, the Cinderella of particle size analysis methods? Powder Technology, p. 115-124, 1979.

LIU, K. Some Factors Affecting Sieving Performance and Efficiency. Powder Technology, 2009.

MILHOMEM, F. O. Modelling of Dewatering in Screens. MSc Dissertation. Ouro Preto, MG, Brazil: Federal University of Ouro Preto, 2013.

RAJA, V. Shale Shaker and Experimental Validation. PhD Thesis. Akron: University of Akron, 2012.

RAJA, V., CHASE, G. G., JONES, B. N. and GEEHAN, T. Computational Modeling and Experiments on Shale Shaker Performance. Proceedings of AADE Fluids Technical Conference and Exhibition. Houston, Texas, 2012.

STANDISH, N. The kinetics of batch sieving. Powder Technology, p. 57-67, 1985.

STANDISH, N., BHARADWAJ, A. K., \& HARIRI-AKBARI, G. A Study of the Effect of Operating Variables on the Efficiency of a Vibrating Screen. Powder Technology, 48, p. 161-172, 1986.

TRUMIC, M., \& MAGDALINOVIC, N. New Model of Screening Kinectics. Minerals Engineering, p. 42-49, 2011.

\section{AGRADECIMENTOS}

Os autores agradecem à UFU, ao CNPq e à PETROBRÁS pelos recursos concedidos e apoio em pesquisas no projeto referente ao termo de cooperação $\mathrm{N}^{\circ}$ 0050.0078502.12.9. 